\title{
COASTAL DIGITAL SURFACE MODEL ON LOW CONTRAST IMAGES
}

\author{
Ana-Maria Rosu ${ }^{\mathrm{a}, *}$, Michel Assenbaum ${ }^{\mathrm{b}, *}$, Ywenn De la Torre ${ }^{\mathrm{a}}$, Marc Pierrot-Deseilligny ${ }^{\mathrm{c}}$ \\ ${ }^{a}$ Bureau de Recherches Géeologiques et Minières, Montpellier, France - (am.rosu, y.delatorre)@brgm.fr \\ ${ }^{\mathrm{b}}$ L'Avion Jaune, Montpellier, France- michel.assenbaum@lavionjaune.fr \\ ${ }^{\mathrm{c}}$ Ecole Nationale des Sciences Géographiques / Institut National de l'Information Géographique et Forestière, France - \\ marc.pierrot-deseilligny@ensg.eu
}

KEY WORDS: UAV, low contrast, DSM, coastal survey, MicMac

\begin{abstract}
:
Coastal sandy environments are extremely dynamic and require regular monitoring that can easily be achieved by using an unmanned aerial system (UAS) including a drone and a photo camera. The acquired images have low contrast and homogeneous texture. Using these images and with very few, if any, ground control points (GCPs), it is difficult to obtain a digital surface model (DSM) by classical correlation and automatic interest points determination approach. A possible response to this problem is to work with enhanced, contrast filtered images. To achieve this, we use and tune the free open-source software MicMac.
\end{abstract}

\section{INTRODUCTION}

Sandy coast is strongly urbanized with harbor facilities, coastal tourism and urban infrastructures. This results in an increase of coastal vulnerability to extreme storms phenomenon which impact beach and dune morphologies and the human facilities. To mitigate coastal hazards, it requires a regular monitoring of this environment in order to better understand and prevent shore morphological changes (De la Torre et al., 2013).

\subsection{Coastal Monitoring Methods}

The most common solutions for coastal monitoring are aerial images taken by airplane (Anders and Byrnes, 1991) and terrestrial differential global positioning system (DGPS) surveying (Baptista et al., 2008). The relatively high cost, low frequency of three to five years for airplane acquisitions, low DGPS spatial coverage, difficulty to access sites such as dunes or river mouths represent some of the factors that restrict the possibility of complete monitoring for a same time and space unit.

\subsection{Why Using an UAS?}

An unmanned aerial system (UAS) including a drone and a photo camera could be a good alternative for coastal monitoring as it offers high frequency, relatively low cost photo-analyzing information on the nature and geometry of the coast (coastline position, dunes' plant cover) and elevation data describing the morphological beach evolution (e.g., erosion, accretion areas) by DSMs difference.

In this article we present a method for working on low contrast images taken by drone on sandy regions. Using these images we obtain DSM using the free open source software MicMac (Pierrot-Deseilligny, 2015). DSMs differences obtained from images at different periods of time allows simple regular monitoring of these extremely dynamic regions.

\subsection{MicMac Software}

MicMac-Apero is free open-source software, distributed under the CeCILL-B license, implemented at IGN (Institut National de l'Information Géographique et Forestière, France). Apero (Aerotriangulation Photogrammetrique Experimentale Relativement Operationelle) is used for images orientation processes and MicMac (Multi-Images Correspondances par Méthodes Automatiques de Corrélation) computes multi-image sub-pixel correlation. MicMac's fields of application include three-dimensional modeling in archeology, architecture for cultural heritage and geology and surveying for environmental applications (Guérin et al., 2012, Lisein et al., 2013), as well as two-dimensional displacement measurements (Rosu et al., 2015).

From now on, we will use the general name MicMac when speaking of MicMac-Apero software.

In this article we present a method for working on low contrast images taken by drone on sandy regions. We have used and tuned MicMac for working with this kind of images.

\section{METHODOLOGY}

\subsection{Field Acquisition}

\subsubsection{Sensors Used}

We used a multirotor UAV that carried a Ricoh GR 16.9 megapixels lightweight digital camera, a Trimble high-precision global navigation satellite system (GNSS) receiver (L1/L2, precise point positioning (PPP) and real-time kinematic (RTK) capable).

\footnotetext{
* am.rosu@brgm.fr

* michel.assenbaum@lavionjaune.fr
} 
Tests were conducted on the Mediterranean coast at Les Baronnets dune-beach system in the Gulf of Lions. The flight altitudes were of $60 \mathrm{~m}$ and $120 \mathrm{~m}$, leading respectively to ground spatial resolution of 1 and $2 \mathrm{~cm}$. Several parallel flight tracks were flown with high overlap, up to $80 \%$ along track and $60 \%$ across track.

For the initial tests, several GCPs were positioned on the ground with centimetric accuracy and materialized by high contrast 12 by $12 \mathrm{~cm}$ targets, which can be used for verification, and ignored when testing the actual correlation methods in development.

\subsubsection{Images Description}

Images acquired by UAV on these sandy environments are low contrast images and have weak textures (some examples are presented in Figure 1).

A low contrast image has a local low ratio between the brightest and the darkest pixel intensities (Rathi et al., 2012).
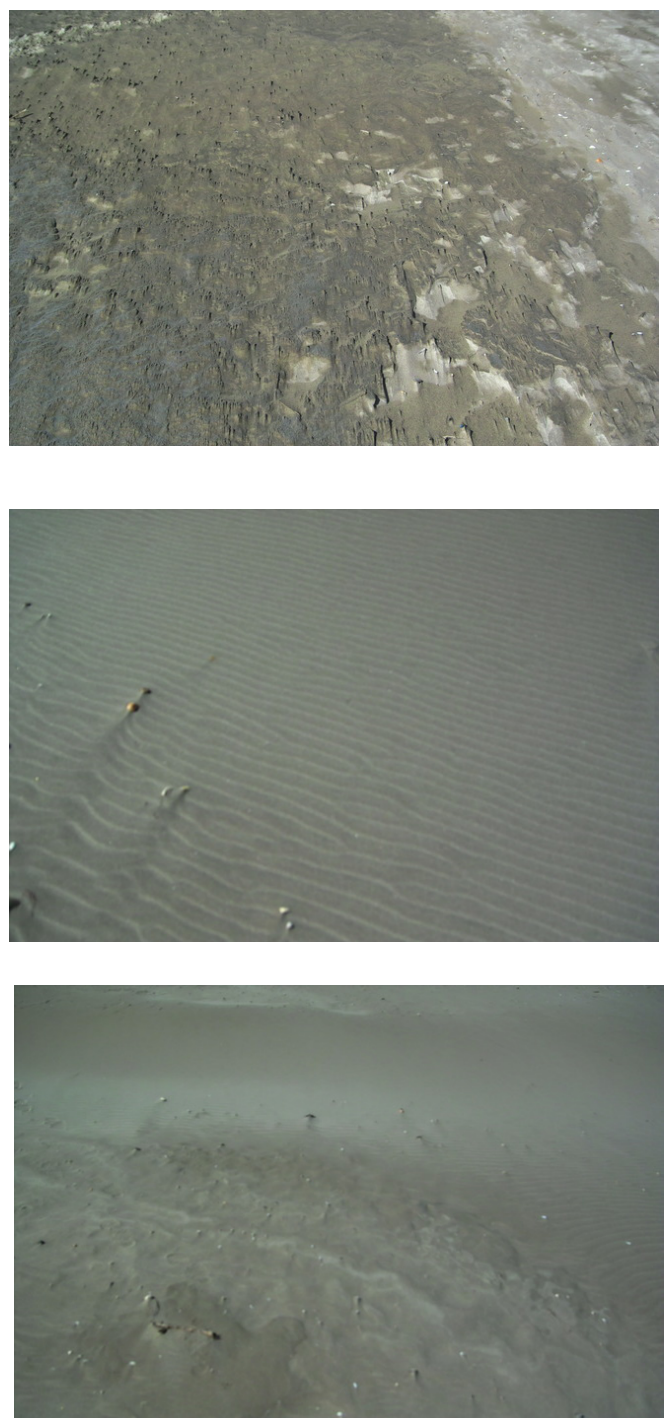

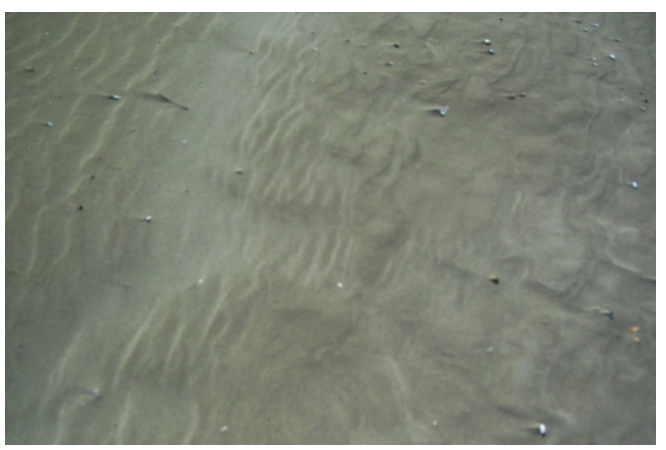

Figure 1. Types of sand textures on Mediterranean coast

Therefore, these images are extremely difficult to match by classical methods.

\subsection{Local Contrast Enhancement Technique}

We used the free open source software MicMac and tuned it in order to be able to obtain satisfactory results using this kind of low contrast images.

MicMac, in its current implementation, uses the SIFT++ algorithm for tie-points detection. SIFT ++ is a lightweight $\mathrm{C}++$ implementation of SIFT detector and descriptor originally created by David Lowe (Lowe, 1999, Lowe, 2004). This implementation produces interest points and descriptors which are very similar to David Lowe's implementation. But, according to Pierrot-Deseilligny (2015), the current implementation of is not fully invariant to scaling or translation in radiometry, which may be a problem when working on low contrast images, as the information may be assimilated to noise and therefore impossible to extract.

If the low contrast images have a good signal-to-noise ratio, there is potential information to compute tie-points. In order to extract all the possible information from these images, we used the raw format for images when available, and a local contrast enhancement based on local statistics (Beghdadi and Le Negrate, 1989; Dale-Jones and Tjahjad, 1993; Zhu and Lam, 1999) before computing tie points. The enhanced images are used only for computing tie-points.

In MicMac, the contrast enhancement is based on local means and standard variation computed for each pixel in a given neighborhood. The function of contrast enhancement is arctan $((x-\mu) / \sigma)$ (where $x$ represents the pixel gray value in the original histogram, $\mu$ and $\sigma$ represent the average gray level and the standard deviation in the 101-by-101 neighborhood around the pixel). The window has to be large enough in order to filter out the noise and small enough in order to be sensitive to small details in the image (Beghdadi and Negrate, 1989).

Figure 2 presents the same image before and after enhancement - details that are difficult to discern on the original image are well visible on the enhanced image. The images presented in Figure 2 are 8-bits images obtained from the same raw image using a MicMac variation of the open-source program dcraw ${ }^{1}$ developed by Dave Coffin for decoding raw image. The information used for obtaining the 8-bits images is the weighted

$1 \mathrm{http} / / /$ www.cybercom.net/ dcoffin/dcraw/ 
sum of the three channels with a weight of 1 for each channel. To obtain the enhanced image, the contrast enhancement algorithm is applied to the image.
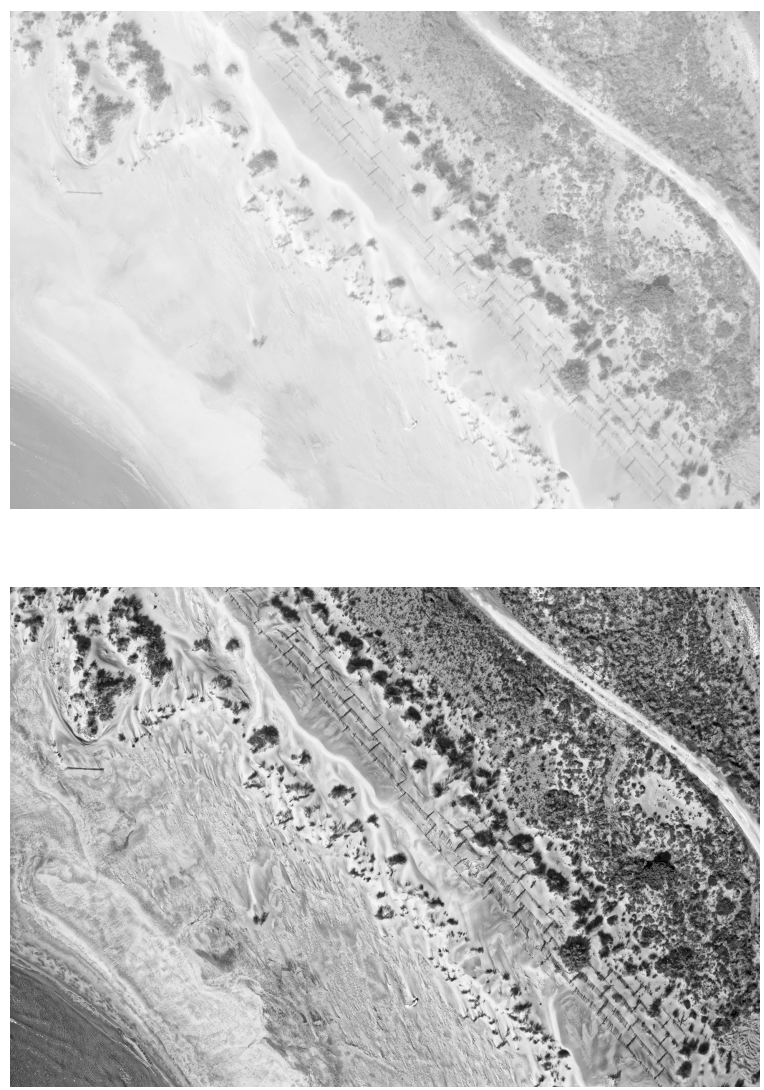

Figure 2. Same image before and after enhancement

According to Hummel (1977), the visual contrast of a digitized image can be improved by adjusting the grayscale so that the histogram of the resulting image is flat. The most desirable histogram output is a flat distibution. This assumption is motivated by the knowledge that a uniform distribution makes best use of the information transmission capabilities of the available gray levels. However, there is no reason to expect the flat histogram to yield a visually optimal version of any image. Histogram flattening can often destroy visual perceptibility of the image.

The histogram equalization is one of the most common techniques for image contrast enhancement. Histogram equalization is equivalent to transforming an image $I_{i}$ into an identity function $H_{i}\left(I_{i}\right)$ on $[0,1]$ (Delon, 2000).

The increase in visual contrast is an artificial enhancement due to a more judicious choice of the relative intensities represented by the quantization levels of the original image. Although the process provides no enhancement in an information-theoretic sense, applying the appropriate transformation can result in a remarkable increase in visual clarity (Hummel, 1977). with and without contrast enhancement, on raw and jpg images to see if the information is valid or if noise prevails.

Raw images are uncompressed - large file size with no loss of data from the camera sensor - images. Jpg images are on the contrary compressed - fairly small in file size with loss of information. Raw images have 12 bits per channel, jpg ones only 8 bits per channel. The dynamic range, defined as a ratio between the smallest and highest pixel intensity, of a raw image is higher than the one of a jpg image.

\subsection{Tie Points}

Tie points were computed with MicMac's implementation of SIFT++ algorithm. The computation is done on original raw and jpg images and on enhanced ones. An example of tie points distribution result is presented in Figure 3. There is a clear improvement concerning the number and the distibution of tie points on enhanced images.
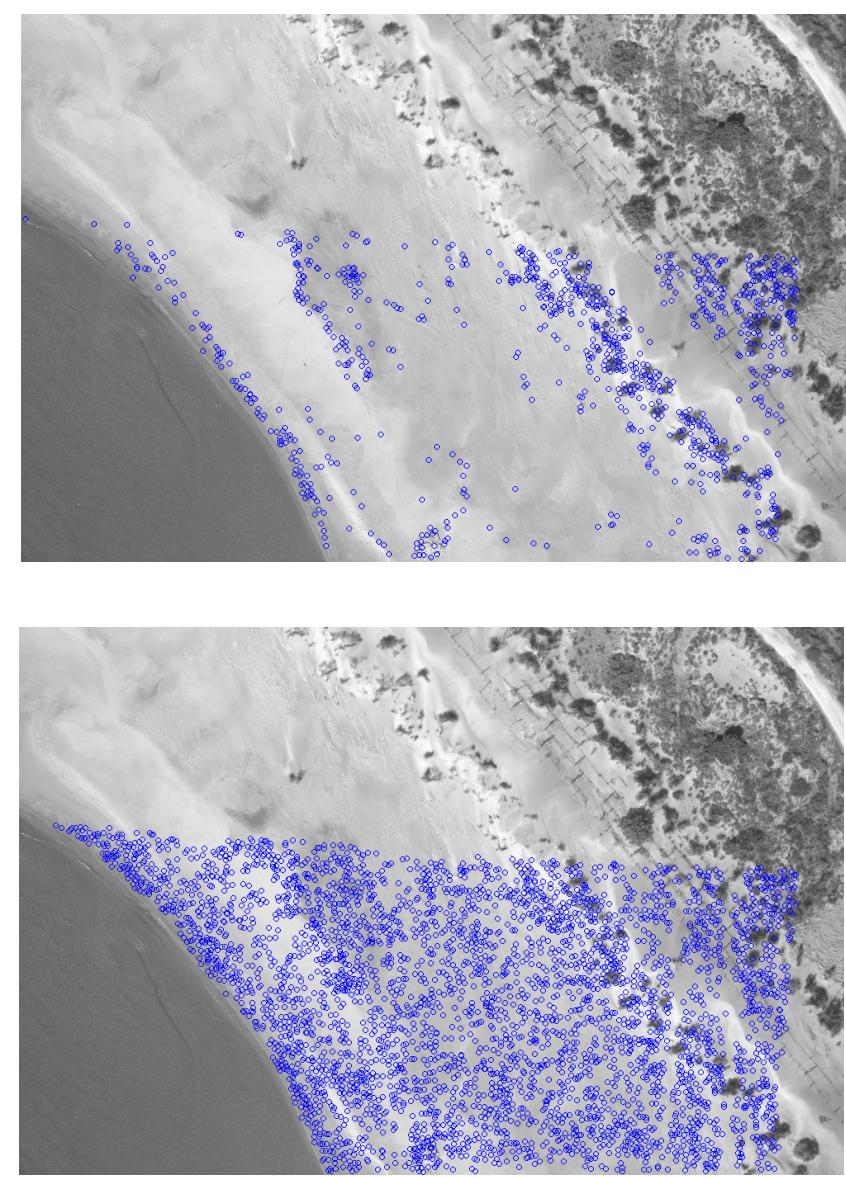

Figure 3. Tie-points distribution on images before and after enhancement

\section{RESULTS}

Contrast enhancement technique may lead overenhancement of noise contrast in the image. Tests were conducted on images 


\subsection{Local Aerotriangulation}

The tie points are used to compute local aerotriangulation. The statistics computed after this process gives information on the number of points used in the computation, their percentage as well as the mean square error.

Table 1 shows the comparison on the number of tie-points found on jpg and raw images with and without contrast enhancement, and the mean squared error for each. For each of the four cases, there is a very high percentage of tie-points used for relative orientation computation, the number of points (simple or multiple tie-points) is almost $50 \%$ higher in the case of contrast enhancement images (jpg or raw) with the highest number for the enhanced raw images. But a high number of tiepoints does not suffice for ensuring good orientation results, the quality of these points being more important than their number. The mean square error quantifies the quality of the tie-points for each case.

\begin{tabular}{|c|l|r|r|r|r|}
\hline Image & MTP & MTP \% & \multicolumn{1}{c|}{ MMTP } & MMTP \% & MSE (px) \\
\hline JPG & 10385.9 & 99.57 & 8407.3 & 99.57 & 0.77 \\
RAW & 15344.3 & 99.69 & 12659.0 & 99.71 & 0.70 \\
CeJPG & 24332.1 & 99.66 & 19092.7 & 99.65 & 0.88 \\
CeRAW & 30598.0 & 99.35 & 24603.8 & 99.76 & 0.80 \\
\hline
\end{tabular}

Table 1. Comparison between results on jpg and raw images with and without contrast enhancement (CeJPG, $C e R A W$ - contrast enhanced JPG and, respectively, RAW images; $M T P$ - mean of number of tie-points per image; $M T P \%$ - mean percentage of used tiepoints; MMTP - mean of multiple tie-points wich are tie-points between more than two images; MSE mean squared error).

In Figure 4 we present tie points clouds obtained with jpg and raw images with and without contrast enhancement. The point clouds obtained using images without enhancement are less dense (barely any point in some homogeneous regions) than the ones using images with contrast enhancement. The density of a point cloud is proportional to the number of tie points used in the computation.

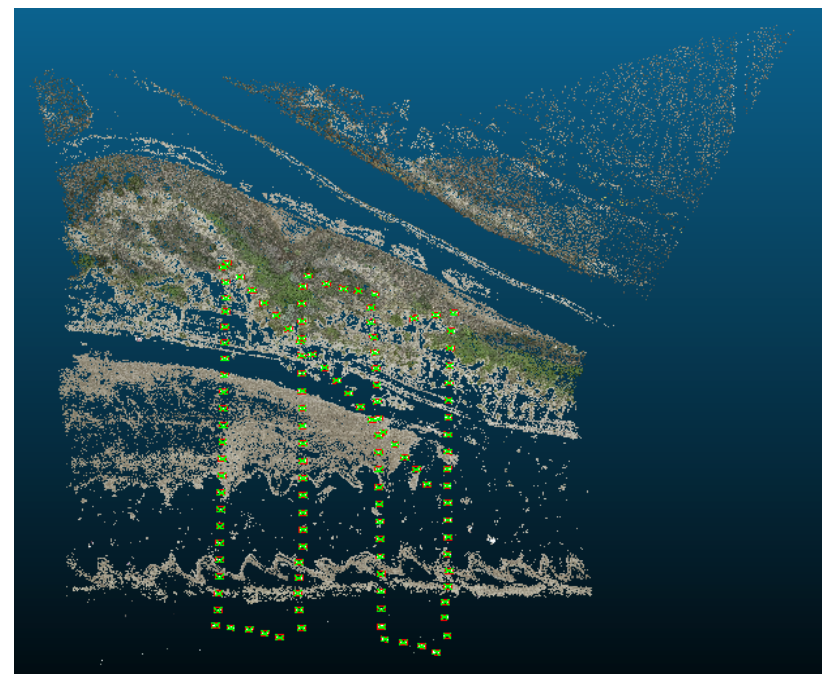

(a)

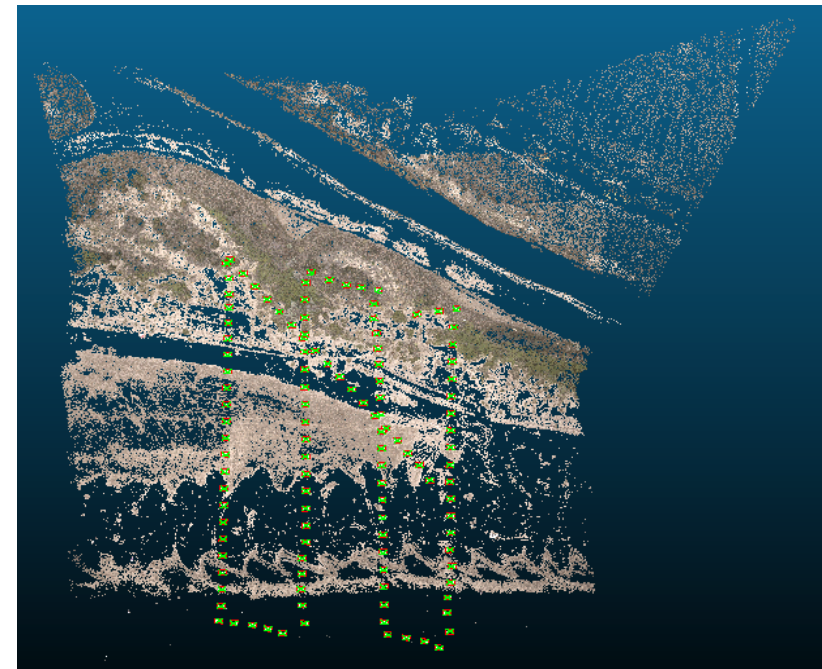

(b)

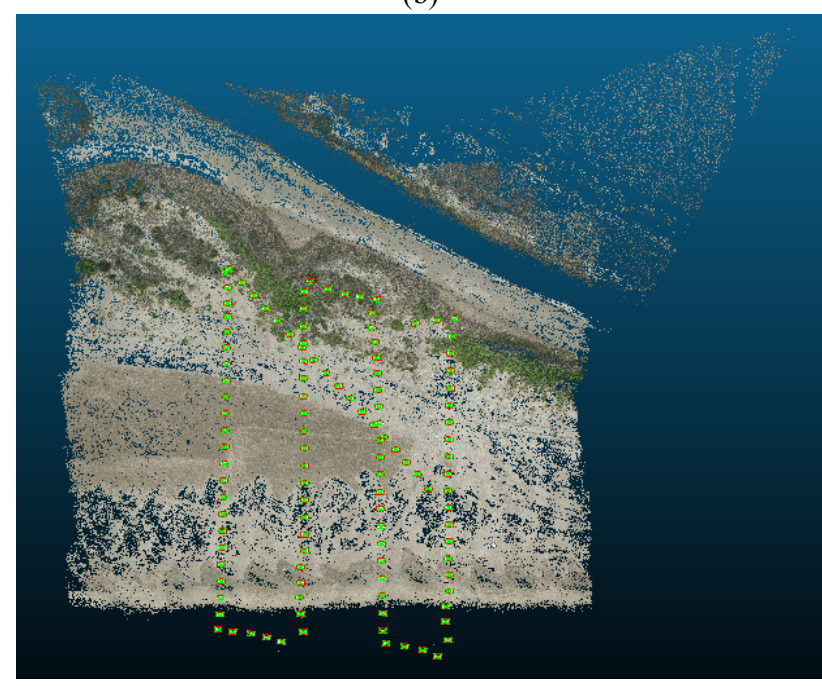

(c)

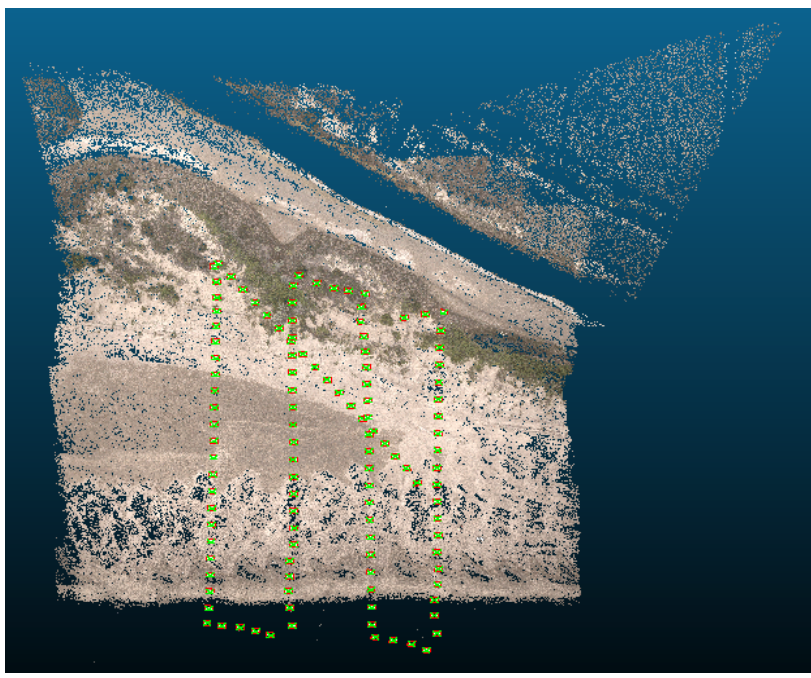

(d)

Figure 4. Point clouds obtained with jpg and raw images with and without contrast enhancement: $4 \mathrm{a}$ - JPG images without enhancement; 4b - RAW images without enhancement; 4c - JPG images with enhancement; $4 \mathrm{~d}-\mathrm{RAW}$ images with enhancement. 


\subsection{Absolute Aerotriangulation / Georeferencing}

Ground control points (GCPs) were used for georeferencing the images. The GCPs were materialized on the ground and independent total station measurements were done on the field with an expected local consistency of $5 \mathrm{~mm}$ in horizontal and vertical.

A simple 7-parameter transformation is applied to the local aerotriangulation. The residuals obtained show the potential deformation between the local aerotriangulation and the field measurements.

The geometric precision obtained after the georeferencing on jpg and raw images with and without contrast enhancement (Table 2) shows that the best precision is obtained when using contrast enhanced raw images when computing tie-points.

\begin{tabular}{|c|r|r|}
\hline Image & Plani $(\mathrm{m})$ & \multicolumn{1}{|c|}{ Alti $(\mathrm{m})$} \\
\hline JPG & 0.0042 & 0.0047 \\
RAW & 0.0037 & 0.0048 \\
CeJPG & 0.0039 & 0.0045 \\
CeRAW & 0.0032 & 0.0041 \\
\hline
\end{tabular}

Table 2. Geometric precision when using jpg and raw images with and without contrast enhancement when computing tie-points (CeJPG, CeRAW - contrast enhanced JPG and, respectively, RAW images; plani, alti - planimetric and altimetric precision)

\section{CONCLUSION}

This project focuses on sandy environments, beaches or dunes. Working with images acquired on these environments involves working with low contrast images. MicMac gives satisfactory results when working with this kind of images, especially when applying a contrast enhancement algorithm on the images before computing tie-points. The comparison between jpg and raw images with and without contrast enhancement shows that the best geometric precision is obtained when working on raw contrast enhanced images.

The next step will be to compute dense DSM. MicMac's dense correlation technique is invariant to local contrast. MicMac uses the original images and not the 8-bits ones.

The current method for georeferencing the images uses GCPs. In a coastal area, especially beaches, there are very few invariant natural GCPs. So for each UAV flight it is required to do DGPS surveying on different GCPs, which increases the costs and limits the interest of using an UAV. Therefore, we want to minimize the use of GCPs. Direct georeferencing (Schwarz et al., 1993, Shukla and Smith, 2000) might represent a valid alternative to this problem. Direct georeferencing most often implies the use of a very high quality GNSS receiver and an INS, which implies load and cost increases. Another solution is to use only the positions of camera center for each shot without attitude measurements, with GNSS only measurements (Turner et al., 2013).

\section{ACKNOWLEDGEMENTS}

The authors would like to thank CAPTIVEN for funding this project. We also thank FUGRO GEOID for performing the ground measurements.

\section{REFERENCES}

Anders, F.J. and Byrnes, M.A., 1991. Accuracy of shoreline change rates as determined from maps and aerial photographs. Shore and Beach, 59(1), 17-26.

Baptista, P., Bastos, L., Bernardes, C., Cunha, T., and Dias, J., 2008. Monitoring sandy shores morphologies by DGPS-a practical tool to generate digital elevation models. Journal of Coastal Research, 24(6), 1516-1528.

Beghdadi, A. and Negrate, A. L., 1989. Contrast Enhancement Technique Based on Local Detection of Edges. Computer Vision, Graphics, and Image Processing, 46, 162-174.

Dale-Jones, R. and Tjahjad, T., 1993. A Study and Modification of the Local Histogram Equalization Algorithm. Computer Vision, Graphics, and Image Processing, 26(9), 1373 - 1381.

De la Torre Y, Balouin Y., Heurtefeux H. and Guérinel B., 2013. The "Storm Network" as a participative network for monitoring the impacts of coastal storms along the littoral zone of the gulf of Lions, France In: Conley, D.C.,Masselink, G., Russell, P.E. and O'Hare, T.J. (eds.), Proceedings 12th International Coastal Symposium (Plymouth,England), Journal of Coastal Research, Special Issue, 65, 927-932.

Delon, J., 2004. Midway Image Equalization. Journal of Mathematical Imaging and Vision, 21, 119-134.

Guérin, C., Binet, R., and Pierrot-Deseilligny, M., 2012. Détection des changements d'élévation d'une scène par imagerie satellite stéréoscopique. In: RFIA 2012 (Reconnaissance des Formes et Intelligence Artificielle), Lyon, France.

Hummel, R., 1977. Image Enhancement by Histogram Transformation. Computer Graphics and Image Processing, 6, 184-195.

Lisein, J., Pierrot-Deseilligny, M., Bonnet, S., and Lejeune, P., 2013. A Photogrammetric Workflow for the Creation of a Forest Canopy Height Model from Small Unmanned Aerial System Imagery. Forests, 4(4), 922-944.

Lowe, D.G., 1999. Object recognition from local scale-invariant features. In: International Conference on Computer Vision, Corfu, Greece, 1150-1157.

Lowe, D.G., 2004. Distinctive image features from scaleinvariant keypoints. International Journal of Computer Vision, 60(2), 91-110.

Pierrot-Deseilligny, M., 2015. MicMac, Apero, Pastis and Other beverages in a Nutshell! http://logiciels.ign.fr/?Micmac.

Rathi, M.S., Karode, A. H., and Suralkar, S.R., 2012. Contrast Enhancement and Smoothing Using Histogram Modification Method for Medical Images. International Journal of Computer Technology and Applications, 3(5), 1789-1798. 
Rosu, A.-M., Pierrot-Deseilligny, M., Delorme, A., Binet, R., and Klinger, Y., 2015. Measurement of ground displacement from optical satellite image correlation using the free opensoure software MicMac. ISPRS Journal of Photogrammetry and Remote Sensing, 100, 48-59.

Schwarz, K., Chapman, M., Cannon, M., and Gong, P., 1993. An Integrated INS/GPS Approach to the Georeferencing of Remotely Sensed Data. Photogrammetric Engineering and Remote Sensing, 59(11), 667-1674.

Shukla, R.K. and Smith, M.J., 2000. Geo-referencing casi imagery using direct measurement of position and attitude. In: International Archives of Photogrammetry and Remote Sensing, XXXIII(Part B2), 502-509.

Turner, D., Lucieer, A., and Wallace, L., 2013. Direct Georeferencing of Ultrahigh- Resolution UAV Imagery. IEEE Transactions on Geoscience and Remote Sensing, 52(5), 27382745.

Zhu, H., Chan, F. H. Y., and Lam, F. K., 1999. Image Contrast Enhancement by Constrained Local Histogram Equalization. Computer Vision and Image Understanding, 73(2), 281-290. 